\title{
16S rRNA gene sequencing and LC-MS-based metabolomics show that ischemic stroke is related to gut microbiota and metabolome in rats
}

\section{Wanfeng Wu}

Henan University of Traditional Chinese Medicine

\section{Yihang Sun}

Henan University of Traditional Chinese Medicine

Shaowu Cheng

Henan University of Traditional Chinese Medicine

Ning Luo

Henan University of Traditional Chinese Medicine

\section{Cheng Cheng}

Henan University of Traditional Chinese Medicine

\section{Chengting Jiang}

Henan University of Traditional Chinese Medicine

HuiFang Nie

Henan University of Traditional Chinese Medicine

\section{Qingping Yu}

Henan University of Traditional Chinese Medicine Jinwen Ge ( $\sim$ xmubinyu@gmail.com )

\section{Research article}

Keywords: ischemic stroke, gut microbiota, metabolomics

Posted Date: October 11th, 2019

DOI: https://doi.org/10.21203/rs.2.15920/v1

License: (c) (1) This work is licensed under a Creative Commons Attribution 4.0 International License.

Read Full License 


\section{Abstract}

\section{Background}

Ischemic stroke (IS) is a common type of stroke with high rates of morbidity, mortality, and disability. Despite accumulating evidence that the gut microbiome and metabolome are associated with human diseases, whether they contribute to the pathophysiological mechanism of IS and whether microbial communities affect metabolic phenotype and function are unclear.

Results

In this study, we integrated 16S rRNA gene sequencing and LC-MS-based metabolomics to explore the roles and underlying mechanisms of the gut microbiome and metabolome in a rat model of IS. Microbiota composition and diversity in IS and control rats were significantly different at the phylum and genus levels. The relative abundance of the phylum Firmicutes was significantly decreased, whereas Proteobacteria and Deferribacteres were markedly increased in IS rats compared with abundance levels in controls. In addition, the metabolic profiles of IS rats were significantly different from those of control rats. We detected 308 significantly dysregulated metabolites, including 155 up-regulated and 153 downregulated, that best distinguished the IS and control groups. Furthermore, correlation analysis revealed that dysbiosis of the gut microbiota was strongly correlated with dysregulated metabolites. Overall, our results showed that IS is characterized by significant alterations in gut microbiota composition and diversity as well as metabolic phenotype.

Conclusion

These results demonstrate that dysbiosis of gut microbiota and perturbations of gut microflora-related metabolites are involved in the development of IS and may serve as potential biomarkers of ischemic stroke.

\section{Background}

Stroke is a disease of sudden interruption of blood supply to the brain and is a common cause of death and disability worldwide. In China, a country with a serious stroke burden, ischemic stroke (IS) accounts for $85 \%$ of all stroke cases and is characterized by high rates of morbidity, mortality, and disability [1, 2]. A large number of studies have suggested that the pathogenesis of IS involves complex and aberrant biological processes, such as energy metabolism disorders, oxidative stress, and the inflammatory response [3-5]. With the extensive application of interventional therapy and deep understanding of the pathophysiological process of ischemic necrosis of brain cells, great achievements have been made in the treatment of IS. However, due to the limited treatment window and high sensitivity of nerve cells to ischemia and hypoxia and their non-reproducibility, the overall efficacy of treatment is still unsatisfactory. Thus, understanding its etiology and finding new biomarkers, drugs, or therapeutic targets to reduce the fatality and disability rates of IS are still urgent problems to be solved. 
Microbiota research, particularly on the gut microflora, has recently gained much attention in medical research. Mounting evidence suggests that dysregulated gut microflora contributes to development of many diseases, such as diabetes, obesity, and inflammatory bowel disease [6-8]. Studies have indicated that the gut microbiota can affect central nervous system physiology and behavior by regulating the microbiota-gut-brain axis. For example, Yu et al. observed in patients with major depressive disorder that depression not only perturbs the gut microbiota at the abundance level but also alters the fecal metabolic phenotype [9]. In recent years, several studies have suggested that dysbiosis of the gut microbiota is associated with IS pathogenesis $[10,11]$. For example, Yamashiro et al. reported that the gut microbiota compositions of patients who suffered strokes were significantly different from those of healthy individuals [11]. Stanley et al. found that commensal bacteria translocated and disseminated in poststroke infection [10]. These observations highlight potential close associations between gut microbiota and IS pathogenesis. However, the specific alterations in the gut microbiota and their effects on key molecular pathways or functions under IS conditions remain unclear and elucidate the possible mechanisms of IS.

It has been suggested that the gut microbiome plays profound roles in modulating host metabolism. Aberrant metabolic functions associated with perturbations in gut microbiome composition play important roles in the development of human diseases $[12,13]$. For example, Wang et al. found that gut microflora-generated trimethylamine $\mathrm{N}$-oxide from dietary choline and carnitine is strongly associated with atherosclerosis in animal models and clinical cohorts [12]. Metabolomics profiling has disclosed aberrant metabolites and metabolic pathways involved in the development of complex diseases [14]. Therefore, explorations of gut microbiome-related metabolic changes associated with perturbed gut microbiota may be particularly useful. Many metabolome studies have been used to unravel metabolic phenotype variations associated with gut microbiota perturbations in disease development $[15,16]$. For instance, Le Gall et al. found altered metabolic activity of the gut microbiota in patients with ulcerative colitis or irritable bowel syndrome [15]. However, given the relationship between the gut microbiota and metabolic phenotype, the consequential questions of how microbial communities influence metabolic function and whether the microbiota and metabolome contribute to IS progression remain unanswered. Therefore, it is very important to assess gut microbiota-related metabolic phenotype alterations, which may advance understanding of the pathophysiological mechanism of IS, and to explore the roles of the gut microbiota in the development of IS and develop potential biomarkers for diagnosis, treatment, and prediction of clinical outcomes in patients with IS.

In the present study, we integrated gut microbiome and metabolomics analysis to explore the roles of gut microbiota and its related metabolic mechanisms in IS. To characterize the composition, dysbiosis, and functions of the gut microbiota in IS, we applied 16S rRNA gene sequencing. To reveal aberrant metabolites and metabolic functions involved in IS, we further performed LC-MS-based metabolomics. Finally, we explored associations between the gut microbiota and metabolism and their contributions to the development of IS. Our results show that IS significantly alters not only gut microbiota composition and diversity but also metabolic phenotype. These results demonstrate that dysbiosis of the gut 
microbiota and perturbations of gut microflora-related metabolites are involved in the development of IS and may serve as potential biomarkers of IS.

\section{Methods}

\section{Animals and samples}

Seven to nine-week-old male Sprague Dawley rats (body weight 240-270 g) raised under specificpathogen-free conditions were purchased from the Hunan SJA Laboratory Animal Co., Ltd. (Changsha, China). A total of 17 rats were housed at the animal center of the First Hospital of Hunan University of Chinese Medicine (Changsha, China) at $25^{\circ} \mathrm{C}, 45-65 \%$ humidity, and with water and food available ad libitum. The rats were used to construct a middle cerebral artery occlusion (MCAO) model of cerebral ischemia-reperfusion injury as previously described $[10,17,18]$. All rats were randomly divided into 2 experimental groups, the IS group and control group. Briefly, all rats were anesthetized by intraperitoneal injection of $10 \%$ chloral hydrate at $0.3 \mathrm{mg} / \mathrm{kg}$ body weight. To induce MCAO, a $10 \mathrm{~mm}$ incision was made on the right-hand side of the neck, and the common carotid artery (CCA), external carotid artery (ECA), and pterygopalatine artery (PPA) were dissected free. Monofilament nylon fishing line of diameter $0.26 \mathrm{~mm}$ was wrapped with paraffin at $0.5 \mathrm{~cm}$ and marked at a distance of $20 \mathrm{~mm}$ across the origin of the midcerebral artery. The monofilament lines were inserted through the right CCA incision, and the PPAs were briefly clipped to prevent misinsertion. The lengths of the suture lines were approximately $18-20 \mathrm{~mm}$ as measured from the bifurcation of the CCA (slightly adjusted according to animal weight). The right middle cerebral artery was embolized, and then the skin was sutured and the tail of the suture was fixed to the skin. Control rats were subjected to a sham operation: they received the initial anesthetic and neck incision only. Animal body temperature was maintained at $37( \pm 0.5)^{\circ} \mathrm{C}$ during ischemia. IS rats exhibiting paralysis of the left limb, exhibiting instability when standing, and lifting the tail to one side after recovery from anesthesia indicated success of the IS model. All rats were given a neurological severity score to assess impairment of neural function in IS as described by Zea et al. [19] $72 \mathrm{~h}$ after establishment of the IS model. The rats were then sacrificed by cervical dislocation. Caecum contents were collected and stored at $-80^{\circ} \mathrm{C}$. Abdominal aortic blood was extracted, and plasma was immediately separated and stored at $-80^{\circ} \mathrm{C}$. The present study was approved by the Ethics Committee of the Hunan University of Traditional Chinese Medicine.

\section{S rRNA amplicon sequencing library preparation}

Genomic DNA was isolated from caecum content using the E. Z. N. A. Stool DNA Kit (Omega Bio-tek, Norcross, GA, USA) according to the manufacturer's protocol. The 16S rDNA V3-V4 region of the ribosomal RNA gene was selected for amplification by PCR $\left(95^{\circ} \mathrm{C}\right.$ for $2 \mathrm{~min}$, followed by 27 cycles at 98 ${ }^{\circ} \mathrm{C}$ for $10 \mathrm{~s}, 62{ }^{\circ} \mathrm{C}$ for $30 \mathrm{~s}$, and $68^{\circ} \mathrm{C}$ for $30 \mathrm{~s}$ and a final extension at $68^{\circ} \mathrm{C}$ for $10 \mathrm{~min}$ ) using primers $341 \mathrm{~F} \mathrm{(CCTACGGGNGGCWGCAG)} \mathrm{and} \mathrm{806R} \mathrm{(GGACTACHVGGGTATCTAAT),} \mathrm{with} \mathrm{an} \mathrm{eight-base} \mathrm{barcode}$ sequence unique to each sample. PCR reactions were performed in triplicate with $50 \mu \mathrm{L}$ mixtures 
containing $5 \mu \mathrm{L}$ of $10 \times$ KOD Buffer, $5 \mu \mathrm{L}$ of $2.5 \mathrm{mM}$ dNTPs, $1.5 \mu \mathrm{L}$ of each primer $(5 \mu \mathrm{M}), 1 \mu \mathrm{L}$ of KOD Polymerase, and $100 \mathrm{ng}$ of template DNA. Amplicons were extracted from $2 \%$ agarose gels and purified using the AxyPrep DNA Gel Extraction Kit (Axygen Biosciences, Union City, CA, USA) according to the manufacturer's instructions and quantified using QuantiFluor-ST (Promega, Madison, WI, USA). After equimolar pooling, the purified amplicons were sequenced on an Illumina platform (Hiseq2500; Illumina, Inc., San Diego, CA, USA) according to standard protocols to generate paired-end reads $(2 \times 250)$.

\section{Analysis of 16S rRNA amplicon sequencing data}

Adapter sequences were removed from the raw data, and low-quality reads were filtered out. Then, pairedend clean reads were merged as raw tags using FLSAH ( $v$ 1.2.11) [20] with a minimum overlap of $10 \mathrm{bp}$ and a mismatch error rate of $2 \%$. To obtain high-quality clean tags, raw tags were filtered using the QIIME (V1.9.1) [21] pipeline under specific filtering conditions [22]. Subsequently, clean tags were searched against a reference database to perform reference-based chimera checking with the UCHIME algorithm. All chimeric tags were removed, leaving effective tags for further analysis. Effective tags were clustered into operation taxonomic units (OTUs) of $\geq 97 \%$ similarity using the UPARSE [23] pipeline. The tag sequence with the highest abundance was selected as the reprehensive sequence within each cluster for taxonomic identification. Representative sequences were classified into organisms by a naive Bayesian model using the RDP classifier (Version 2.2) [24] based on the Greengenes Database [25]. Furthermore, biomarker features in each group were screened by the LEfSe software. Alpha diversity indices (including Chao1 and Simpson), OTU rarefaction curves, and rank abundance curves were calculated or plotted in QIIME. Significance of differences in alpha index comparisons was calculated by a Welch's $t$-test in R. In addition, multivariate statistical techniques, including PCA and PCOA, and the analysis of similarities (ANOSIM) test were run with R. Finally, the functional groups of OTUs were inferred using PICRUST (v1.0) [26].

\section{Plasma metabolomics using LC-MS}

We extracted metabolites from plasma samples. Briefly, the samples were thawed at $4{ }^{\circ} \mathrm{C}$ on ice. Then, $100 \mu \mathrm{L}$ of a sample was placed in an Eppendorf tube and extracted with $300 \mu \mathrm{L}$ of methanol. Internal standards $(10 \mu \mathrm{L})$ were added, and the tube was vortexed for $30 \mathrm{~s}$, treated with ultrasound for $10 \mathrm{~min}$ in ice water, and incubated for $1 \mathrm{~h}$ at $-20^{\circ} \mathrm{C}$ to precipitate proteins. The tube was then centrifuged at 12000 rpm for $20 \mathrm{~min}$ at $4^{\circ} \mathrm{C}$, and the supernatant $(200 \mu \mathrm{L})$ was transferred into a fresh $2 \mathrm{~mL}$ glass LC/MS vial. For quality control samples, $10 \mu \mathrm{L}$ aliquots from each sample were pooled. Sample supernatant $(150 \mu \mathrm{L})$ was also used for UHPLC-QTOF-MS analysis. LC-MS/MS analyses were performed using a UHPLC system (1290; Agilent Technologies, Santa Clara, CA, USA) with a UPLC BEH Amide column (1.7 $\mu \mathrm{m}$ 2.1*100 mm; Waters Corp., Milford, MA, USA) coupled to TripleTOF 5600 system (AB Sciex LLC, Framingham, MA, USA). The mobile phase consisted of $25 \mathrm{mM} \mathrm{NH} 4 \mathrm{OAc}$ and $25 \mathrm{mM} \mathrm{NH} 4 \mathrm{OH}$ in water ( $\mathrm{pH}$ $=9.75)(A)$ and acetonitrile $(B)$ and was applied in an elution gradient as follows: 0 min, 95\% B; 0.5 min, 
95\% B; $7 \mathrm{~min}, 65 \%$ B; $9 \mathrm{~min}, 40 \%$ B; $9.1 \mathrm{~min}, 95 \% \mathrm{~B}$; and $12 \mathrm{~min}, 95 \% \mathrm{~B}$, delivered at $0.5 \mathrm{~mL} / \mathrm{min}$. The injection volume was $2 \mu \mathrm{L}$. A Triple TOF mass spectrometer was used for its ability to acquire MS/MS spectra on an information-dependent basis (IDA) during the LC/MS experiment. In this mode, the acquisition software (Analyst TF 1.7, AB Sciex) continuously evaluates the full-scan survey MS data as it is collected and triggers the acquisition of MS/MS spectra depending on preselected criteria. In each cycle, 12 precursor ions whose intensities were greater than 100 were chosen for fragmentation at a collision energy (CE) of $30 \mathrm{~V}$ (15 MS/MS events with product ion accumulation time of $50 \mathrm{~ms}$ each). ESI source conditions were set as following: Ion source gas 1 as 60 Psi, lon source gas 2 as 60 Psi, Curtain gas as $35 \mathrm{Psi}$, source temperature $650^{\circ} \mathrm{C}$, Ion Spray Voltage Floating (ISVF) $5000 \mathrm{~V}$ or $-4000 \mathrm{~V}$ in positive and negative modes, respectively.

\section{Computational analysis of metabolomics data}

MS raw data (.raw) files were converted to the mzML format using ProteoWizard and processed by the XCMS R package (version 3.2) [27], which includes retention time alignment, peak detection, and peak matching. Each sample was subsequently normalized to an internal standard. The preprocessing results generated a data matrix that consisted of retention time (RT), mass-to-charge ratio (m/z), and peak intensity values. The CAMERA R package was used for peak annotation after XCMS data processing. An in-house MS2 database was applied in metabolite identification. Furthermore, multivariate statistical analysis, including PCA, partial least squares-discriminant analysis (PLS-DA), and orthogonal projection to latent structures-discriminant analysis (OPLS-DA) were performed for metabolite profiling. To identify differential metabolites, a variable importance in projection (VIP) score in the OPLS model was applied to rank the metabolites that best distinguished between two groups. The threshold for VIP was set to 1. In addition, Student's $t$-test was also used as a univariate analysis for screening differential metabolites. Those with $p$-values $<0.05$ and VIP $\geq 1$ were considered differential metabolites between two groups. Pathway enrichment analysis identified significantly enriched metabolic pathways for differential metabolites.

\section{Correlations between the gut microbiome and metabolome}

To integrate microbiota and metabolomic data, we performed an O2PLS analysis. O2PLS models were constructed using the metabolite abundance dataset and each taxonomy level of the microbiota dataset, including the phylum and genus levels. The 02PLS models were build using the OmicsPLS package of R [28]. In addition, to explore correlations between the microbiota and metabolome, Pearson correlation coefficients between the phylum and genus levels of the microbiota and metabolomic datasets were calculated in $\mathrm{R}$ (version 3.5.1). The correlation heatmap was generated using the pheatmap package in $\mathrm{R}$, and network analysis was performed using the igraph package in $\mathrm{R}$. 


\section{Results}

\section{Changes in the gut microbiome of rats with IS}

A diagram of the experiment and workflow of 16S rRNA gene sequencing and metabolite profiling used to investigate the impact of the gut microbiome and metabolism in IS is shown in Figure 1. Firstly, we created a model of cerebral ischemia in rats and found that the neurological severity scores in the group receiving the MCAO operation were higher than those in control group, suggesting successful establishment of the IS model (Supplementary Table 1). Then, the V3-V4 region of the 16S rRNA gene amplicon was sequenced from caecum samples from a total 17 rats, including 9 from the IS group and 8 from the control group. The average sequencing depth obtained for the 17 rats was 114409 reads per sample (Supplementary Table 2). Untargeted metabolomics (LC-MS/MS) were performed on plasma samples for metabolite profiling of IS. To assess relationships between microbiota and metabolites, we integrated microbiome and metabolome data.

To investigate differences in microbiota composition between the IS and control group, we performed bioinformatics analysis on the 16s rRNA gene sequencing data. The sequences from IS model samples with $>97 \%$ similarity at the OTU level were distinct from control samples, implying differences in community composition between the two groups (Figure 2A and Supplementary Figure 1A). Hierarchical clustering analysis by the unweighted pair group method with arithmetic mean (UPGMA) also showed clear differences in the IS model and control rats (Supplementary Figure 1B). A total of 10 phyla were identified by $16 \mathrm{~s}$ rRNA gene sequencing. Bacteroidetes, Firmicutes, and Proteobacteria were the three main components of the gut microbiome, and different samples showed different relative abundances of the phyla (Figure 2B and Supplementary Figure 1C, D). Additionally, we observed that the alpha diversity metrics (Ace, Chao1, Shannon, and Simpson's diversity indices) of IS rats were slightly higher than those of control rats, although not significantly so (Figure 2C; Supplementary Figure 1E, F; and Supplementary Table 3), which may indicate differences between the richness and diversity of gut microbiota in the IS model and control rats. To further demonstrate community differences between the two groups, we performed an ANOSIM based on the Bray-Curtis distance across samples. We found significant community differences between the IS model and control groups $(R=0.553, p$-value $=0.001$, Figure $2 D)$.

To explore specific changes in the gut microbiome caused by IS, we applied Welch's $t$ test to identify significant differences between phyla abundance levels in the IS model and control rat groups. The abundance levels of three phyla, namely, Firmicutes, Proteobacteria, and Deferribacteres, were significantly different in the two groups (Figure 3A, B, and C). The abundance of Firmicutes was significantly higher in the control than IS group $(p=0.0196$, Figure $3 C)$, whereas Proteobacteria and Deferribacteres were both significantly higher in the IS than control group $(p=0.033$ and $p=0.021$, respectively). Furthermore, 14 genera exhibited significantly changed abundance levels between the IS and control groups (Supplementary Figure 2A, B). Additionally, differential species were determined by the LEfSe method, which is used to analyze differences between the microflora of groups and find the predominant species in each group [29]. We found that the abundance levels of two species, namely, 
Ruminococcus_sp_15975 (LDA score $=2.77, \mathrm{p}$-value $=0.0005)$ and Lachnospiraceae_UCG_001 (LDA score $=2.308$, $p$-value $=0.0003$ ), were both significantly different between the two groups (Supplementary Figure 2C). Notably, LDA scores indicated that Ruminococcus_sp_15975 and Lachnospiraceae_UCG_001 may be biomarker microflora specific to the IS and control groups, respectively.

To investigate functional changes in the gut microbiota, we performed functional predictions using the Tax4Fun software [30]. We found that some KEGG pathways, such as Amino acid metabolism, Transcription, Replication and repair, and Neurodegenerative diseases, were significantly changed in the two groups (Figure 3DE). For example, we observed a remarkable up-regulation of the Amino acid metabolism and Energy metabolism pathways in the IS group. On the other hand, the Translation and replication and Repair pathways were down-regulated in the IS group. These results suggest that microbial alterations may have contributed to functional shifts in the IS rats. In summary, the composition, diversity, and functions of the gut microbiome in the IS rats showed differences from those of the control rats, suggesting that the gut microbiome plays key roles in the development of IS.

\section{Perturbations in the metabolome of the gut microbiome in IS rats}

Dysbiosis of the gut microbiota reportedly impacts metabolism [31]. Metabolic perturbations may correlate with changes observed in the gut microbiome and reflect its functional changes. Hence, we examined the plasma metabolome of the rats using untargeted metabolic profiling (LC-MS) to determine the putative effects of altered microbial composition on metabolite profiles. In the OPLS-DA model, there was a much bigger difference between the metabolic profiles of the control and IS rats for metabolites in the positive and negative modes (Figure $4 \mathrm{AB}$ and Supplementary Figure 3), suggesting that IS may lead to significant biochemical changes and that there are potential biomarkers in the different groups. To identify differentially abundant metabolites in the IS and control groups, a VIP score was applied to the OPLS model to rank metabolites that best distinguished between the two groups. We detected 159 and 149 significantly dysregulated metabolites in the positive and negative modes, respectively (Figure 4C, D; Supplementary Figure 4A; Supplementary Table 4 and 5). For metabolites in the positive mode, 86 were up-regulated and 73 down-regulated in the IS group compared to those in the control group. For metabolites in the negative mode, 69 were up-regulated and 80 down-regulation in the IS group compared to those in the control group. As shown in Figure 4CD, the abundance levels of differential metabolites exhibited significantly different patterns in the IS and control groups (Supplementary Figure 4A). For example, POS7164 (FDR $=0.0014$, VIP score $=3.64$ ) was up-regulated 3.5 -fold in the IS group comparing with that in the control group, and POS2928 (FDR $=0.0012$, VIP score $=1.33$ ) was down-regulated 4-fold in the IS group compared with that in the control group (Figure 4E). NEG4099 (FDR $=0.01$, VIP score $=$ 1.73) was up-regulated 4.1 -fold in the IS group compared to the control group, and NEG1229 (FDR $=0.02$, VIP score $=3.13)$, annotated to 3-indolepropionic acid, was down-regulated 7.5-fold in the IS group 
compared to the control group (Figure 4F). These results suggest that these dysregulated metabolites may be involved in the development of IS. To further investigate the functional changes induced by the dysregulated metabolites, the metabolites were mapped to KEGG metabolic pathways for pathway and enrichment analysis. We found that many metabolites were annotated with KEGG metabolism-related pathways. For example, 22 metabolites were annotated with Amino acid metabolism, and 22 with Lipid metabolism (Supplementary Figure 4B). In addition, 2 metabolites were involved in Neurodegenerative diseases. Moreover, KEGG pathway enrichment analysis showed that the dysregulated metabolites were significantly enriched with Cholinergic synapse, mTOR signaling pathway, 2-oxocarboxylic acid metabolism, and other associations (Supplementary Figure 5). These data suggest that the metabolite profiles in the IS group differed from those in the control group, and significantly dysregulated metabolites were involved in metabolic or synaptic processes, suggesting that perturbations in the metabolome play key roles in the development of IS.

\section{Correlations between the gut microbiome and metabolites}

The impact of the microbiome on metabolic activity has been extensively studied [31-33]. Therefore, we integrated the microbiome and metabolomic datasets with 02PLS analysis to infer relationships between differential metabolites and phyla during IS. The 02PLS model captured co-variation between the gut microbiome and metabolites $\left(R^{2} X=0.47, R^{2} Y=0.542, R^{2} X\right.$ corr $=0.43, R^{2} Y$ corr $\left.=0.456\right)$, and the variations in phyla correlated with those in the metabolome, showing substantial overlap (Figure $5 \mathrm{~A}$ and $B$, Supplementary Figure $6 A, B, C$ ) and suggesting that perturbations in the microbiome influence metabolite profiles. As shown in Figure $5 A$ and $B, 10$ metabolites were significantly correlated with certain phyla. Furthermore, to explore the functional relationships between gut microbiome perturbations and altered metabolite levels, a correlation matrix was generated with Pearson's correlation coefficient. We observed significant correlations between the gut microbiome and altered metabolite levels $(r>0.5$ or $r<-$ $0.5, p<0.05)$. Interestingly, strong correlations were found between specific gut bacteria and differential metabolites (Figure 5C, Supplementary Figure 6D), which may indicate the presence of gut microbiotarelated metabolites. For example, abundance of Proteobacteria was significantly higher in IS than control rats (Figure $3 \mathrm{C}$ ) and was significantly positively correlated with POS1742 (fold change $=2.23, \mathrm{p}$-value $=$ 0.009 ), POS7164 (fold change $=3.51, \mathrm{p}$-value $=0.00003$ ), NEG4099 (fold change $=4.11, \mathrm{p}$-value $=$ 0.0006 ), and NEG13345 (fold change $=3.29$, p-value $=0.006$; Figure $5 \mathrm{C}$ ). Meanwhile, Proteobacteria were also significantly negatively correlated with POS2928 (fold change $=-4.11, \mathrm{p}$-value $=0.00002$ ) and POS8452 (fold change $=-2.61$, p-value $=0.00098$; Figure 5C). Additionally, the lower abundance of Firmicutes in IS was significantly positively correlated with POS2928, NEG1179 (indoleacrylic acid, fold change $=-4.75, \mathrm{p}$-value $=0.006$ ), and NEG1229 (3-indolepropionic acid, fold change $=-7.46, \mathrm{p}$-value $=$ 0.003 ) and negatively correlated with POS7164 and POS12605 (fold change $=2.30$, p-value $=0.00006$ ). Therefore, gut microflora-related metabolites that were highly correlated with specific gut bacteria 
demonstrated functional correlations between the gut microbiome and metabolites, implying that the gut microbiota influences metabolites involved in IS.

To further exhibit the relationships between the gut microbiome and metabolites, we constructed a correlation network from significantly related phylum/genus-metabolite pairs (Figure 5D and Supplementary Figure 6E). As shown in Figure 5D, a metabolite can relate to multiple phyla, and a phylum can correlate with multiple metabolites, suggesting complex functional relationships between the gut microbiome and metabolome. In summary, the IS model induced significant taxonomic perturbations in the gut microbiome, which in turn may have substantially altered the metabolomic profile of the gut microbiome, as evidenced by changes in various gut microflora-related metabolites.

\section{Discussion}

The etiology of ischemic stroke is complex and heterogeneous, and few biomarkers and therapeutic targets have been used in clinical practice. In this study, we used high-throughput 16S rRNA gene sequencing and metabolomics profiling to investigate the impacts of ischemic stroke on the gut microbiome and metabolic profiles. By integrating microbiome and metabolome data, the results demonstrate significant differences in phyla and genera abundance levels in the gut microbial communities and perturbations in the metabolic profiles of the IS rats. Furthermore, the altered gut microbiota significantly correlated with plasma metabolite levels, suggesting that IS not only alters the gut microbiota but also affects metabolic phenotype, resulting in dysbiosis of the gut microbiota and disorder of metabolite homeostasis. These findings may reveal novel etiologies of IS, reveal the roles of gut microbiota and metabolites in IS, and allow monitoring of the gut microbiota and metabolites as biomarkers or lead to strategies that alter them as therapeutic tools.

It has been shown that IS affects the distribution of the gut microbiota. Wang et al. found that there were differences between diversity and relative abundance levels at the phylum level in the gut microbiota of patients with cerebral infarction and healthy subjects, and Proteobacteria increased while Bacteroidetes decreased in the former group [34]. In our study, 16S rRNA amplicon sequencing revealed similar changes in microbiota composition and major phyla (Proteobacteria and Bacteroidetes) in IS model and control rats. Moreover, three phyla (Firmicutes, Proteobacteria, and Deferribacteres) exhibited significantly different abundance levels between the two groups. Proteobacteria abundance was significantly higher in the IS than in the control group, which was consistent with the results of Wang et al. [34]. However, we also found that the abundance of Firmicutes was significantly higher in the control than in the IS group. Additionally, a novel phylum, Deferribacteres, was also significantly more abundant in the IS group (Figure 3C). PCoA and hierarchical clustering analysis by the UPGMA of OTUs with $>97 \%$ similarity both revealed that the community composition of the IS group was different from that of the control group. Alpha and beta diversity indices showed community differences between the two groups. KEGG pathways, such as Amino acid metabolism, Transcription, and Neurodegenerative diseases, were significantly changed in two groups suggesting that alterations in the gut microbiota induce the dysfunction that characterizes IS. Furthermore, we also observed significantly different abundance levels of 14 genera between the two 
groups (Supplementary Figure 2A, B), such as increased Escherichia-Shigella and decreased Lactobacillus abundances in IS rats, which was consistent with the results of Yamashiro et al [35]. Additionally, the abundance levels of Ruminococcus_sp_15975 and Lachnospiraceae_UCG_001 significantly changed between the two groups as identified by LEfSe (Supplementary Figure 2C). Notably, the LDA scores of Ruminococcus_sp_15975 (LDA score $=2.77)$ and Lachnospiraceae_UCG_001 ((LDA score $=2.308$ ) indicated that the two genera may be microflora-specific biomarkers in the IS and control groups, respectively. However, there were other altered genera that have no reported association with IS. Therefore, further studies to explore the roles of these bacteria in the development of IS and their potential as biomarkers are warranted.

Accumulated evidence has demonstrated that dysbiosis of the gut microbiota influences metabolism, and metabolic perturbations correlate with altered gut microbiota and reflect functional changes to the gut microbiome in human disease $[15,16,31]$. Therefore, to shed light on these putative changes to the metabolome induced by changes in the gut microbiome, we performed untargeted metabolic profiling (LC-MS). Our results revealed that the metabolic profiles of the IS rats were different from those of control rats through multivariate statistical analyses, such as OPLS-DA and PLS-DA. Moreover, we identified 308 significantly dysregulated metabolites that best distinguished between the two groups and that may serve as potential metabolite biomarkers for IS. For example, it is known that indoleacrylic acid promotes intestinal epithelial barrier function and mitigates inflammatory responses in patients with irritable bowel disease [36]. Meanwhile, 3-indolepropionic acid (NEG1229, FDR $=0.02$, VIP score $=3.13$ ) and indoleacrylic acid (NEG1179, FDR $=0.006$, VIP score $=2.73$ ) levels showed 7.5-fold and 4.75 -fold decreases, respectively, in IS rats compared with levels in control rats (Figure 4F), which suggests that the decreasing abundance of indoleacrylic acid may also play key roles in IS. In addition, cholic acid and deoxycholic acid levels showed 13.45 -fold and 3.7-fold decreases, respectively, in IS rats, which is consistent with the observation that patients with coronary artery disease have significantly decreased bile acid excretion levels and less deoxycholic acid than do healthy subjects [37]. Moreover, the dysregulated metabolites were significantly enriched with Cholinergic synapse, mTOR signaling pathway, 2-Oxocarboxylic acid metabolism, and other associations according to KEGG analysis (Supplementary Figure 5). In a previous study, the mTOR cell signaling pathway was crucial to the long-term protective effects of ischemic post-conditioning against stroke [38]. Taken together, our results show that the metabolite profiles of the IS rats differed from those of control rats, perturbations of the metabolome may play key roles in the development of IS, and significantly dysregulated metabolites may serve as important biomarkers of IS after further validation.

Gut microbiota perturbations associated with metabolic phenotype changes are useful for understanding mechanisms in the development of human diseases [31, 39]. Our results indicate that gut microbiota phyla or genera are significantly correlated with metabolite profiles as determined by O2PLS and Pearson's correlation analysis. We identified 10 metabolites significantly correlated with certain phyla, which may be gut microbiota-related metabolites. For example, the phylum Proteobacteria was significantly positively correlated with POS1742, POS7164, NEG4099, and NEG13345. Additionally, strong correlations were found between specific genera and differential metabolites (Supplementary Figure 6). 
For example, indoleacrylic acid was positive correlated with Ruminococcaceae_UCG-009 (correlation efficient $=0.64$ ), Lachnospiraceae_UCG-001 (correlation efficient $=0.62$ ), and Lactobacillus (correlation efficient $=0.68$ ). These findings suggest that the gut microbiome of the IS rats exhibited significant taxonomic perturbations that substantially altered its metabolomic profile, as evidenced by changes in various gut microflora-related metabolites. Therefore, dysbiosis of the gut microbiota and perturbations of gut microflora-related metabolites were involved in the development of IS. Further studies are needed to determine whether these alterations may serve as biomarkers of IS.

\section{Conclusions}

In this study, 16S rRNA gene sequencing and LC-MS based metabolomics were integrated to investigate the relationship between the gut microbiota and its metabolic phenotype on IS. Our results show that IS significantly altered not only the gut microbiota composition and diversity, but also perturbed its metabolic phenotype. Furthermore, correlation analysis revealed that some changes in the gut microbiota strongly correlated with dysregulated metabolites. These findings indicate that the gut microbiota and metabolome are both involved in the pathology of IS, and that IS not only disturbs the gut microbiota at the abundance level, but also alters metabolic homeostasis. Overall, dysbiosis of the gut and dysregulation of gut microbiota-related metabolites may be potential biomarkers for probing the functional effects of IS and developing disease diagnosis, prevention, and therapeutic strategies.

\section{Abbreviations}

Analysis of similarities (ANOSIM)

Common carotid artery (CCA)

Common carotid artery (CCA)

Ischemic stroke (IS)

Middle cerebral artery occlusion (MCAO)

Operational taxonomic unit (OTU)

Pterygopalatine artery (PPA)

\section{Declarations}

\section{Ethics approval}

All the MenSCs and UCMSCs were obtained with the informed consent of the donors and all studies meet the "Declaration of Helsinki". The present study was approved by the Ethics Committee of the Hunan University of Traditional Chinese Medicine. 


\section{Availability of data and materials}

The datasets used and/or analyzed during the current study are available from the corresponding author on reasonable request.

\section{Consent for publication}

Not applicable

\section{Competing interests}

The authors declare that they have no competing interests.

\section{Funding}

This study was supported by the National Natural Science Foundation of China (No. 81774174) and Postgraduate Innovation Project of Hunan University of Chinese Medicine (No. 2018CX01).

\section{Authors Contributions}

The study design was approved by Jinwen Ge and Shaowu Cheng, acquisition of data by Wanfeng Wu,Yihang Sun, Qingping Yu, analysis and interpretation of data by Wanfeng Wu,Yihang Sun,Ning Luo,Cheng Cheng, statistical analysis by Huifang Nie, Chengting Jiang,Wanfeng Wu, drafting the manuscript by Wanfeng Wu, revision of manuscript for important intellectual content by Shaowu Cheng,Jinwen Ge.

\section{Acknowledgements}

Not Applicable

\section{References}

1.Feng SQ, Aa N, Geng JL, Huang JQ, Sun RB, Ge C, et al. Pharmacokinetic and metabolomic analyses of the neuroprotective effects of salvianolic acid $A$ in a rat ischemic stroke model. Acta Pharmacol Sin. 2017;38(11):1435-44 [doi: 10.1038/aps.2017.114] [Pubmed:28836583].

2.Chen Junmin SD, Xiaoxia L, Xiaoyun C, Qian S, Xianjian Z. Advances on cerebrovascular disease researches in 2016. Clin Focus. 2017;32(2). 
3.Rodrigo R, Fernández-Gajardo R, Gutiérrez R, Matamala JM, Carrasco R, Miranda-Merchak A, Feuerhake W. Oxidative stress and pathophysiology of ischemic stroke: novel therapeutic opportunities. CNS Neurol Disord Drug Targets. 2013;12(5):698-714 [doi: 10.2174/1871527311312050015] [Pubmed:23469845].

4.Narne P, Pandey V, Phanithi PB. Interplay between mitochondrial metabolism and oxidative stress in ischemic stroke: an epigenetic connection. Mol Cell Neurosci. 2017;82:176-94 [doi:

10.1016/j.mcn.2017.05.008] [Pubmed:28552342].

5.Yan T, Chopp M, Chen J. Experimental animal models and inflammatory cellular changes in cerebral ischemic and hemorrhagic stroke. Neurosci Bull. 2015;31(6):717-34 [doi: 10.1007/s12264-015-1567-z] [Pubmed:26625873].

6.Ley RE, Bäckhed F, Turnbaugh P, Lozupone CA, Knight RD, Gordon JI. Obesity alters gut microbial ecology. Proc Natl Acad Sci U S A. 2005;102(31):11070-5 [doi: 10.1073/pnas.0504978102] [Pubmed:16033867].

7.Qin J, Li Y, Cai Z, Li S, Zhu J, Zhang F, et al. A metagenome-wide association study of gut microbiota in type 2 diabetes. Nature. 2012;490(7418):55-60 [doi: 10.1038/nature11450] [Pubmed:23023125].

8.Franzosa EA, Sirota-Madi A, Avila-Pacheco J, Fornelos N, Haiser HJ, Reinker S, et al. Gut microbiome structure and metabolic activity in inflammatory bowel disease. Nat Microbiol. 2019;4(2):293-305 [doi: 10.1038/s41564-018-0306-4] [Pubmed:30531976].

9.Jiang $\mathrm{H}$, Ling Z, Zhang Y, Mao H, Ma Z, Yin Y, et al. Altered fecal microbiota composition in patients with major depressive disorder. Brain Behav Immun. 2015;48:186-94 [doi: 10.1016/j.bbi.2015.03.016] [Pubmed:25882912].

10.Stanley D, Mason LJ, Mackin KE, Srikhanta YN, Lyras D, Prakash MD, et al. Translocation and dissemination of commensal bacteria in post-stroke infection. Nat Med. 2016;22(11):1277-84 [doi: 10.1038/nm.4194] [Pubmed:27694934].

11.Xu N, Kan P, Yao X, Yang P, Wang J, Xiang L, Zhu Y. Astragaloside IV reversed the autophagy and oxidative stress induced by the intestinal microbiota of AIS in mice. J Microbiol. 2018;56(11):838-46 [doi: 10.1007/s12275-018-8327-5] [Pubmed:30353470].

12.Wang Z, Klipfell E, Bennett BJ, Koeth R, Levison BS, Dugar B, et al. Gut flora metabolism of phosphatidylcholine promotes cardiovascular disease. Nature. 2011;472(7341):57-63 [doi: 10.1038/nature09922] [Pubmed:21475195].

13.Quinn RA, Adem S, Mills RH, Comstock W, DeRight Goldasich L, Humphrey G, et al. Neutrophilic proteolysis in the cystic fibrosis lung correlates with a pathogenic microbiome. Microbiome. 2019;7(1):23 [doi: 10.1186/s40168-019-0636-3] [Pubmed:30760325]. 
14.Steffens DC, Wei Jiang, Krishnan KR, Karoly ED, Mitchell MW, O'Connor CM, Kaddurah-Daouk R. Metabolomic differences in heart failure patients with and without major depression. J Geriatr Psychiatry Neurol. 2010;23(2):138-46 [doi: 10.1177/0891988709358592] [Pubmed:20101071].

15.Le Gall G, Noor SO, Ridgway K, Scovell L, Jamieson C, Johnson IT, et al. Metabolomics of fecal extracts detects altered metabolic activity of gut microbiota in ulcerative colitis and irritable bowel syndrome. J Proteome Res. 2011;10(9):4208-18 [doi: 10.1021/pr2003598] [Pubmed:21761941].

16.Jansson J, Willing B, Lucio M, Fekete A, Dicksved J, Halfvarson J, et al. Metabolomics reveals metabolic biomarkers of Crohn's disease. PLOS ONE. 2009;4(7):e6386 [doi:

10.1371/journal.pone.0006386] [Pubmed:19636438].

17.Connolly ES, Jr, Winfree CJ, Stern DM, Solomon RA, Pinsky DJ. Procedural and strain-related variables significantly affect outcome in a murine model of focal cerebral ischemia. Neurosurgery.

1996;38(3):523-31; discussion 532 [doi: 10.1097/00006123-199603000-00021] [Pubmed:8837805].

18.Wang W, Wang Q, Yu W, Chen L, Li Z. Efficacy of phosphocreatine pre-administration on XIAP and Smac in ischemic penumbra of rats with focal cerebral ischemia reperfusion injury. Acta Cir Bras. 2018;33(2):117-24 [doi: 10.1590/s0102-865020180020000003] [Pubmed:29513810].

19.Longa EZ, Weinstein PR, Carlson S, Cummins R. Reversible middle cerebral artery occlusion without craniectomy in rats. Stroke. 1989;20(1):84-91 [doi: 10.1161/01.str.20.1.84] [Pubmed:2643202].

20.Magoč T, Salzberg SL. FLASH: fast length adjustment of short reads to improve genome assemblies. Bioinformatics. 2011;27(21):2957-63 [doi: 10.1093/bioinformatics/btr507] [Pubmed:21903629].

21.Caporaso JG, Kuczynski J, Stombaugh J, Bittinger K, Bushman FD, Costello EK, et al. QIIME allows analysis of high-throughput community sequencing data. Nat Methods. 2010;7(5):335-6 [doi: 10.1038/nmeth.f.303] [Pubmed:20383131].

22.Bokulich NA, Subramanian S, Faith JJ, Gevers D, Gordon JI, Knight R, et al. Quality-filtering vastly improves diversity estimates from Illumina amplicon sequencing. Nat Methods. 2013;10(1):57-9 [doi: 10.1038/nmeth.2276] [Pubmed:23202435].

23.Edgar RC. UPARSE: highly accurate OTU sequences from microbial amplicon reads. Nat Methods. 2013;10(10):996-8 [doi: 10.1038/nmeth.2604] [Pubmed:23955772].

24.Wang Q, Garrity GM, Tiedje JM, Cole JR. Naive Bayesian classifier for rapid assignment of rRNA sequences into the new bacterial taxonomy. Appl Environ Microbiol. 2007;73(16):5261-7 [doi: 10.1128/AEM.00062-07] [Pubmed:17586664].

25.DeSantis TZ, Hugenholtz P, Larsen N, Rojas M, Brodie EL, Keller K, et al. Greengenes, a chimerachecked 16S rRNA gene database and workbench compatible with ARB. Appl Environ Microbiol. 2006;72(7):5069-72 [doi: 10.1128/AEM.03006-05] [Pubmed:16820507]. 
26.Langille MG, Zaneveld J, Caporaso JG, McDonald D, Knights D, Reyes JA, et al. Predictive functional profiling of microbial communities using 16S rRNA marker gene sequences. Nat Biotechnol. 2013;31(9):814-21 [doi: 10.1038/nbt.2676] [Pubmed:23975157].

27.Smith CA, Want EJ, O'Maille G, Abagyan R, Siuzdak G. XCMS: processing mass spectrometry data for metabolite profiling using nonlinear peak alignment, matching, and identification. Anal Chem. 2006;78(3):779-87 [doi: 10.1021/ac051437y] [Pubmed:16448051].

28.Bouhaddani SE, Uh HW, Jongbloed G, Hayward C, Klarić L, Kiełbasa SM, Houwing-Duistermaat J. Integrating omics datasets with the OmicsPLS package. BMC Bioinformatics. 2018;19(1):371 [doi: 10.1186/s12859-018-2371-3] [Pubmed:30309317].

29.Segata N, Izard J, Waldron L, Gevers D, Miropolsky L, Garrett WS, Huttenhower C. Metagenomic biomarker discovery and explanation. Genome Biol. 2011;12(6):R60 [doi: 10.1186/gb-2011-12-6-r60] [Pubmed:21702898].

30.Aßhauer KP, Wemheuer B, Daniel R, Meinicke P. Tax4Fun: predicting functional profiles from metagenomic 16S rRNA data. Bioinformatics. 2015;31(17):2882-4 [doi: 10.1093/bioinformatics/btv287] [Pubmed:25957349].

31.Kamada N, Seo SU, Chen GY, Núñez G. Role of the gut microbiota in immunity and inflammatory disease. Nat Rev Immunol. 2013;13(5):321-35 [doi: 10.1038/nri3430] [Pubmed:23618829].

32.Nicholson JK, Holmes E, Kinross J, Burcelin R, Gibson G, Jia W, Pettersson S, hosts. Host-gut microbiota metabolic interactions. Science. 2012;336(6086):1262-7 [doi: 10.1126/science.1223813] [Pubmed:22674330].

33.Li M, Wang B, Zhang M, Rantalainen M, Wang S, Zhou H, et al. Symbiotic gut microbes modulate human metabolic phenotypes. Proc Natl Acad Sci U S A. 2008;105(6):2117-22 [doi: 10.1073/pnas.0712038105] [Pubmed:18252821].

34.Wang W, Li X, Yao X, Cheng X, Zhu Y. The characteristics analysis of intestinal microecology on cerebral infarction patients and its correlation with apolipoprotein E. Med (Baltimore). 2018;97(41):e12805 [doi: 10.1097/MD.0000000000012805] [Pubmed:30313111].

35.Yamashiro K, Tanaka R, Urabe T, Ueno Y, Yamashiro Y, Nomoto K, et al. Gut dysbiosis is associated with metabolism and systemic inflammation in patients with ischemic stroke. PLOS ONE. 2017;12(2):e0171521 [doi: 10.1371/journal.pone.0171521] [Pubmed:28166278].

36.Wlodarska M, Luo C, Kolde R, d'Hennezel E, Annand JW, Heim CE, et al. Indoleacrylic acid produced by commensal Peptostreptococcus species suppresses inflammation. Cell Host Microbe. 2017;22(1):2537.e6 [doi: 10.1016/j.chom.2017.06.007] [Pubmed:28704649]. 
37. Charach G, Grosskopf I, Rabinovich A, Shochat M, Weintraub M, Rabinovich P. The association of bile acid excretion and atherosclerotic coronary artery disease. Ther Adv Gastroenterol. 2011;4(2):95-101 [doi: 10.1177/1756283X10388682] [Pubmed:21694811].

38.Wang P, Xie R, Cheng M, Sapolsky R, Ji X, Zhao H. The mTOR cell signaling pathway is crucial to the long-term protective effects of ischemic postconditioning against stroke. Neurosci Lett. 2018;676:58-65 [doi: 10.1016/j.neulet.2018.03.062] [Pubmed:29605662].

39.Arrieta MC, Stiemsma LT, Dimitriu PA, Thorson L, Russell S, Yurist-Doutsch S, et al. Early infancy microbial and metabolic alterations affect risk of childhood asthma. Sci Transl Med. 2015;7(307):307ra152 [doi: 10.1126/scitranslmed.aab2271] [Pubmed:26424567].

\section{Figures}

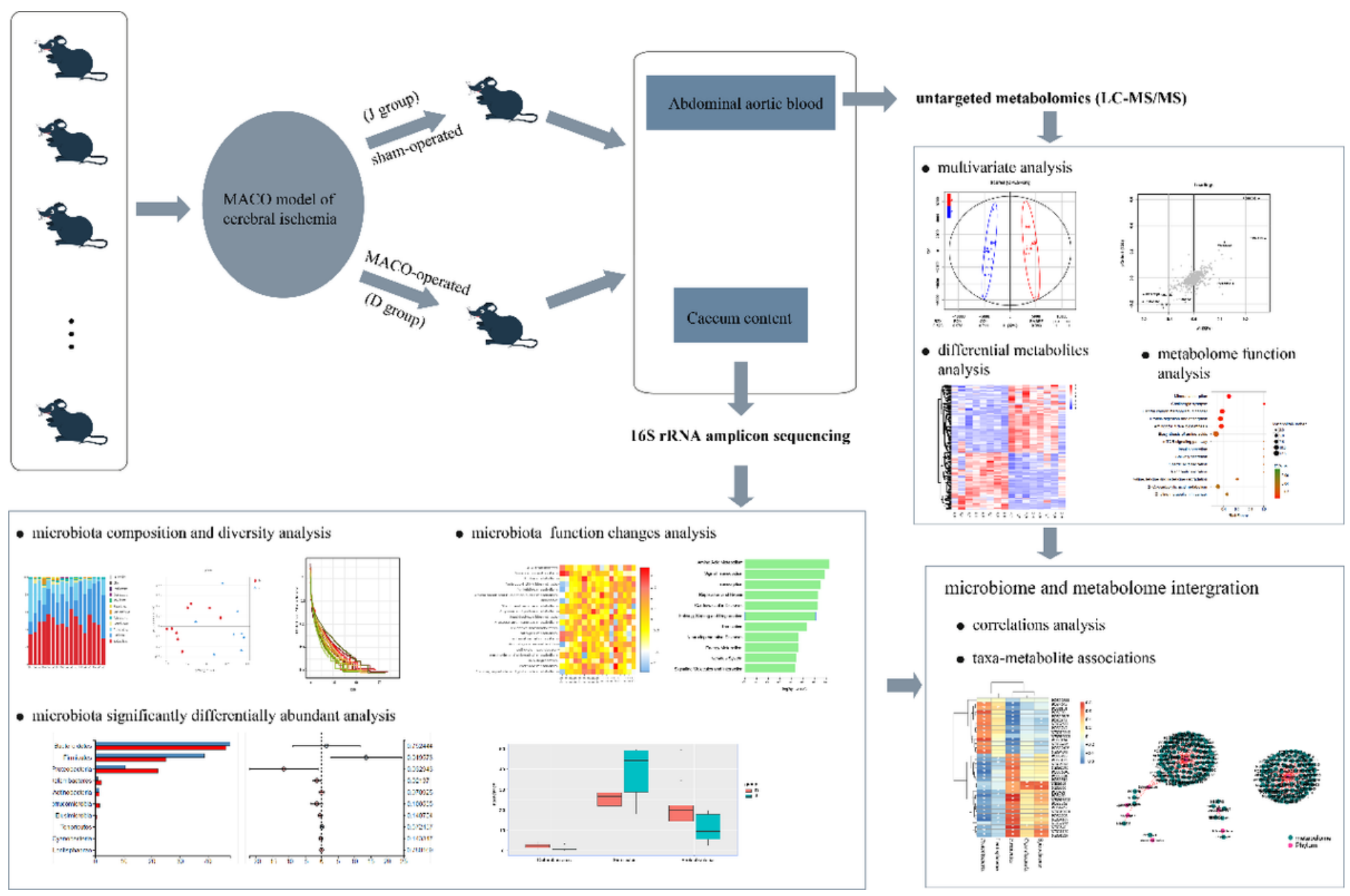

\section{Figure 1}

The experiment and analytical workflow of the study. A total 17 rats were randomly divided into 2 experimental groups, the IS group and control group, and the IS group underwent MCAO. Then, caecum contents were collected for 16S rRNA gene sequencing and metabolomics (LC-MS/MS). Based on the 
16S rRNA amplicon data, composition, and diversity, significantly differentially abundant levels and functional changes in the microbiota were analyzed at the phylum/genus levels. Then, identification of differential metabolites in plasma and prediction of their functions were performed using untargeted metabolomics (LC-MS/MS). Finally, integration of microbiome and metabolome data was employed to analyze relationships between microbiota and the metabolites.

$\Lambda$

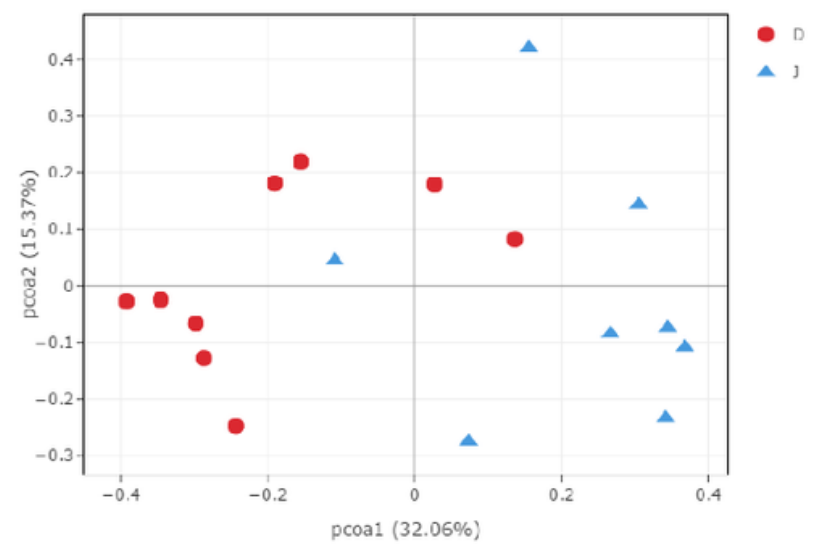

$\mathrm{C}$

Rank Abundance Distribution Curves

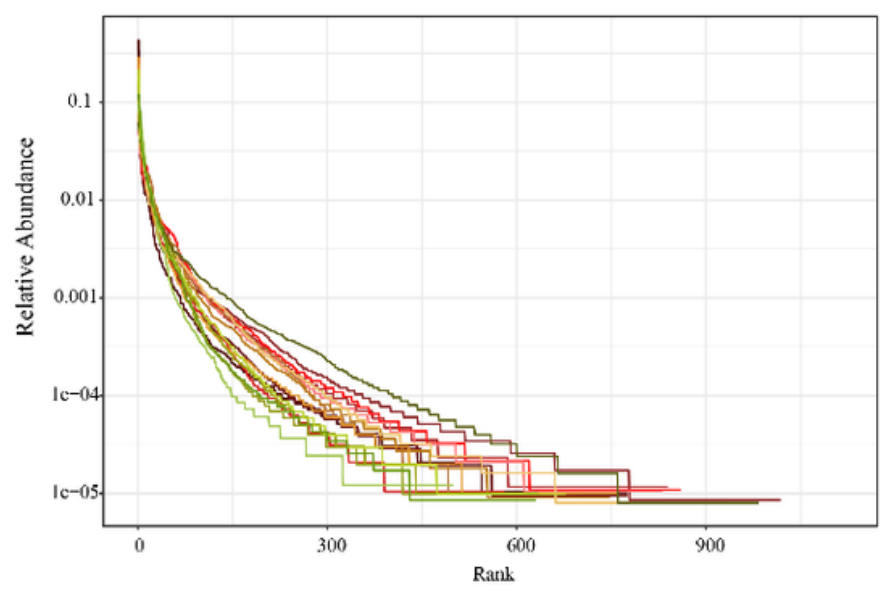

B
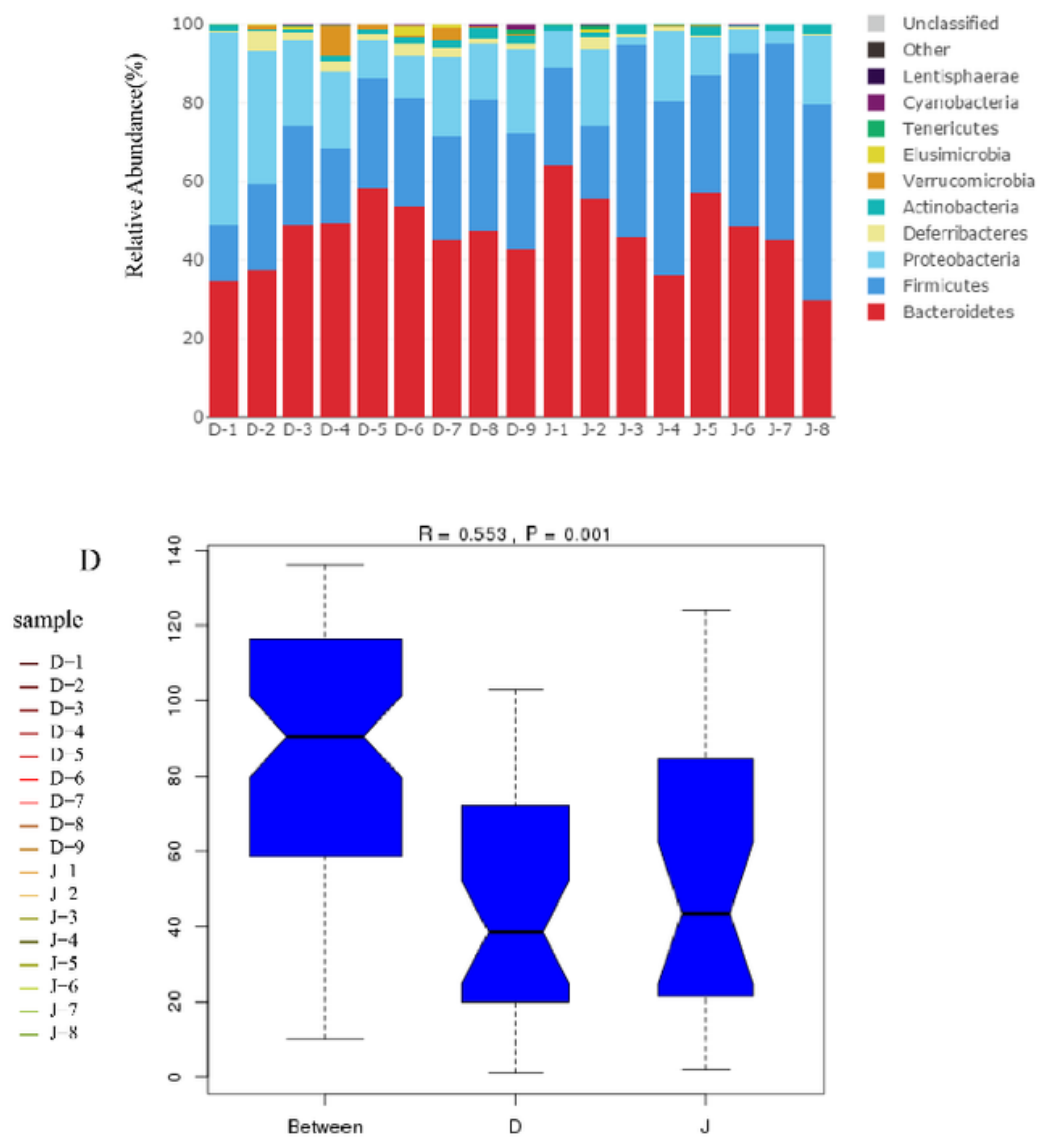

Figure 2

Composition and diversity of gut microbiota. (A) Gut microbiome patterns of IS and control rats differentiated by principal coordinate analysis (PCoA) based on Bray-Curtis distance. (B) Gut microbiome composition profiles at the phylum level in control and IS rats as revealed by $16 \mathrm{~S}$ rRNA sequencing (each color represents one bacterial family). (C) The rank abundance distribution curves of all rats. (D) ANOSIM of IS and control rats. 
A

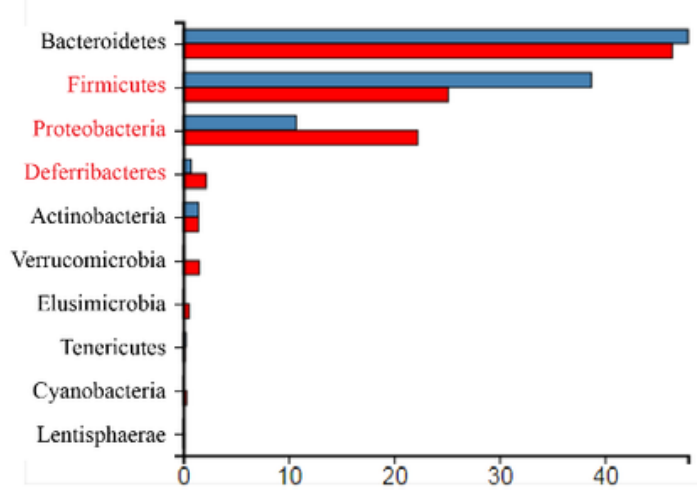

C

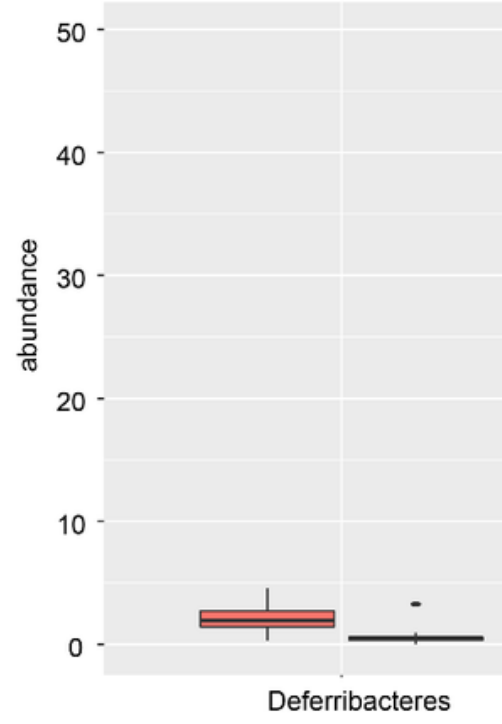

$\mathrm{D}$

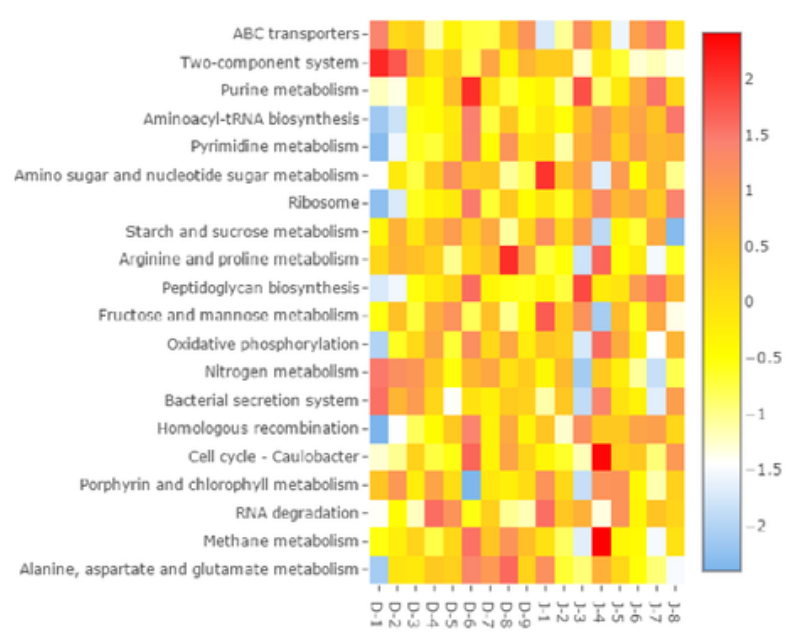

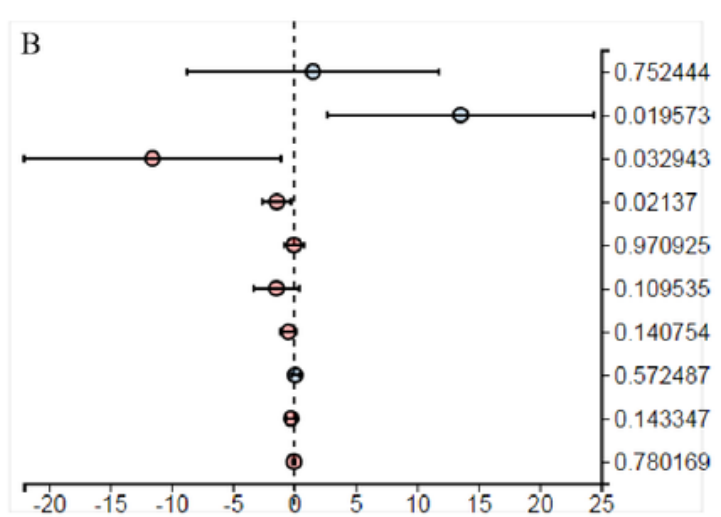
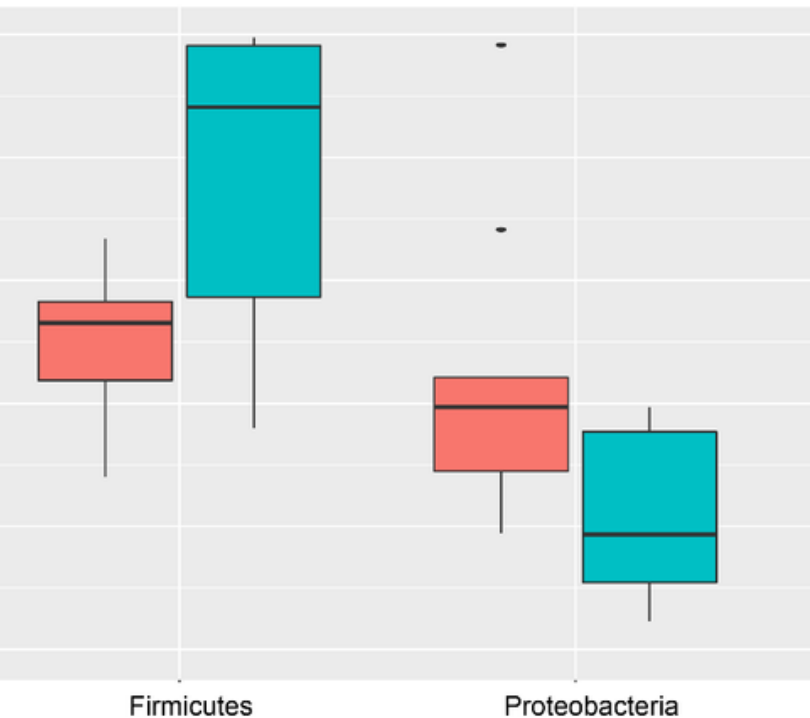

group

官 D

E

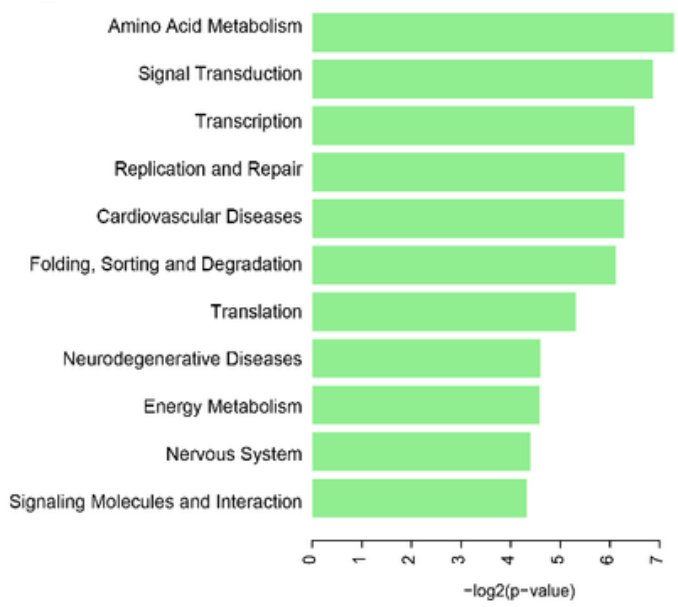

\section{Figure 3}

Significantly different phyla abundance levels and functional associations of gut microbiota in the IS model and control groups. (A) Phylum abundance levels in the gut microbiome of IS and control rats (blue: J group, red: D group). (B) Significance of differences in phylum abundance levels of the gut microbiome in (A). (C) The abundance levels of three significant differentially phyla in IS and control rats. 
(D) Heatmap plot of the abundance of predicted KEGG pathways by Tax4Fun in all samples. (E) Differential KEGG functions identified by Welch's t-test between two groups.

$\wedge$

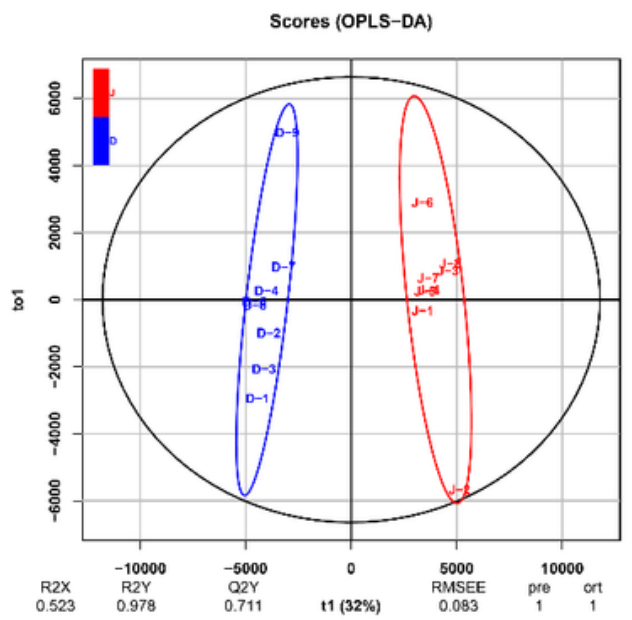

B

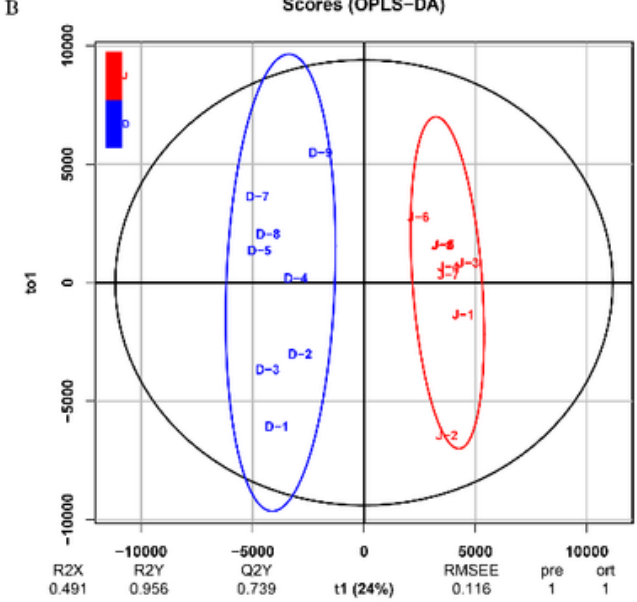

c
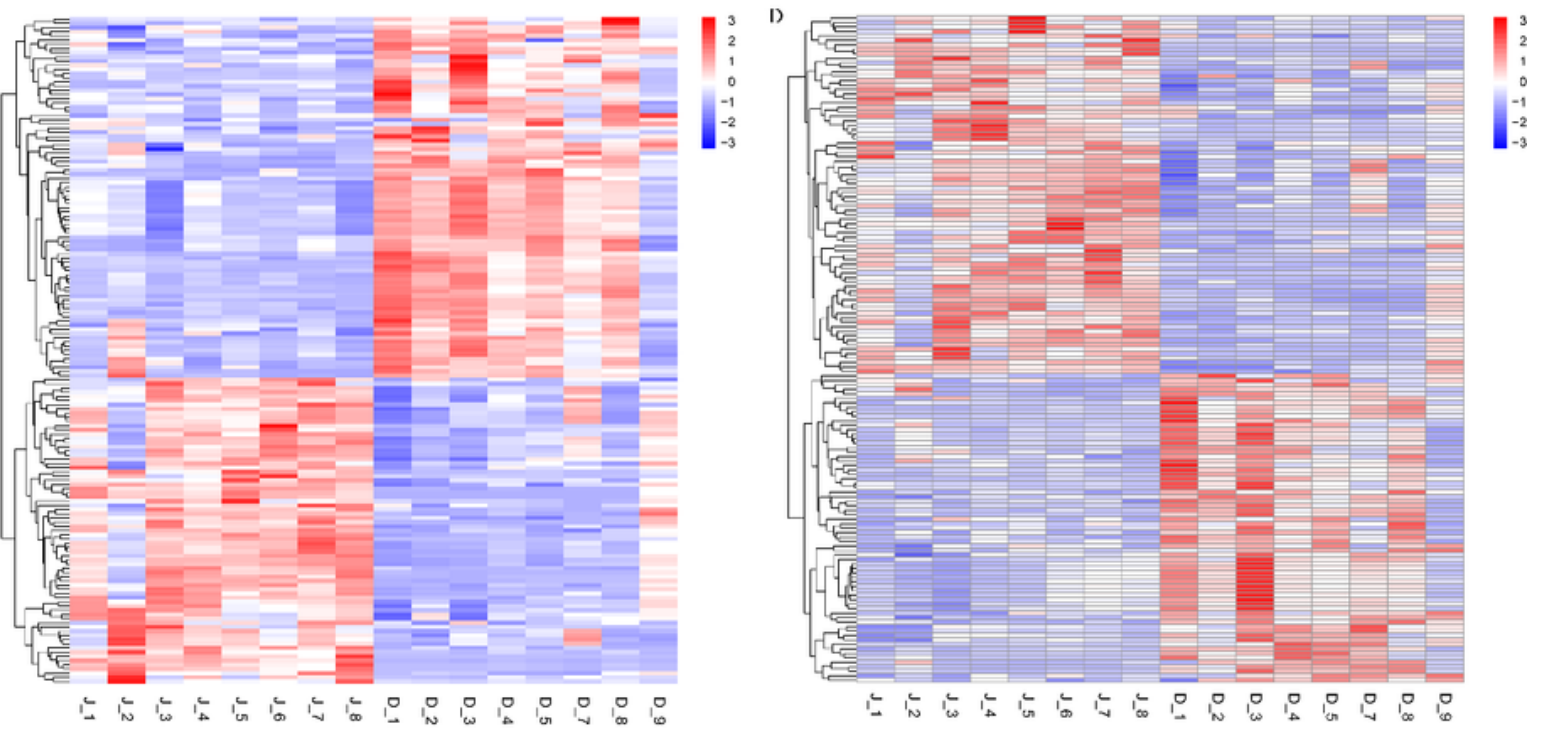

L
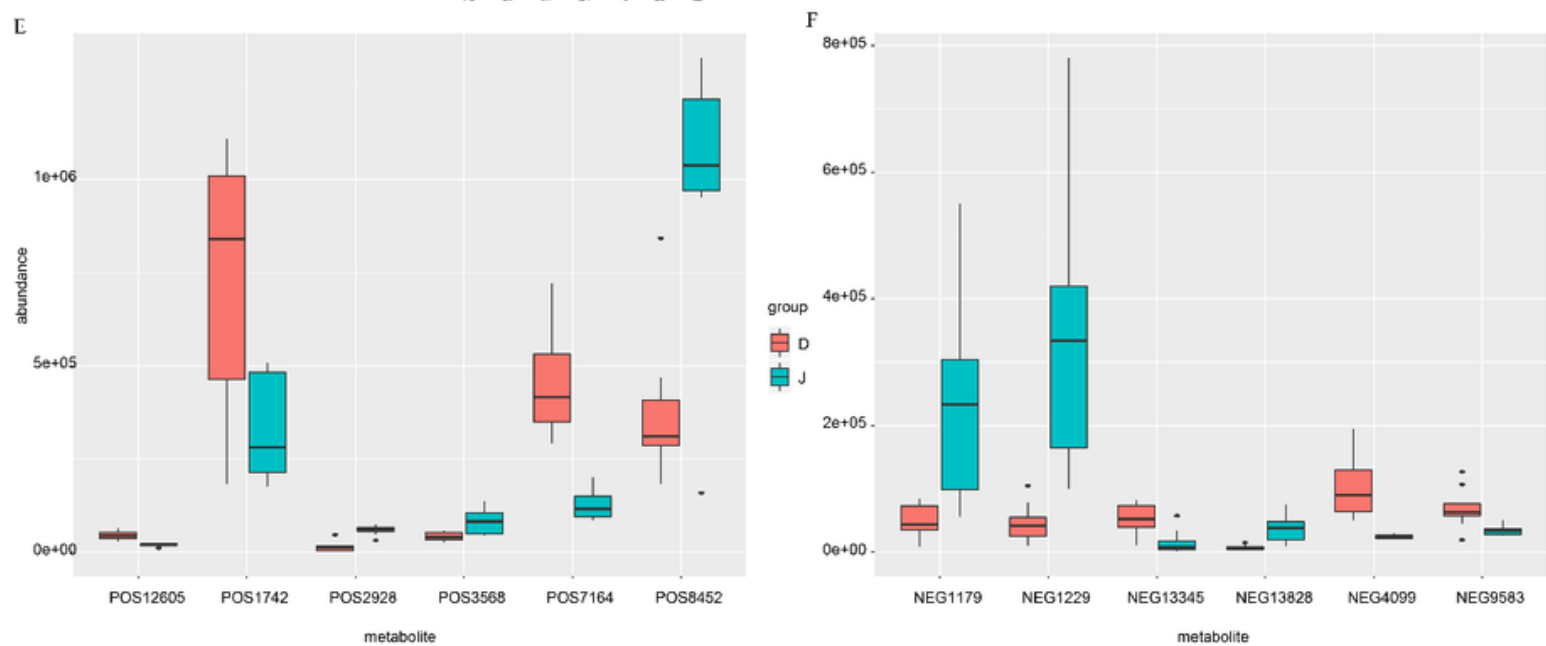

Figure 4

Significantly differential metabolites in the IS and control groups. (A and B) The OPLS-DA score plots of blood samples from the control and IS groups for positive ion mode (A) and negative ion mode (B) of metabolites. (C and D) Heatmap plot of significantly altered metabolites in positive ion mode (C) and 
negative ion mode (D). (E and F) Abundance of significantly altered metabolites in positive ion mode (E) and negative ion mode (F) across two groups. NEG1229 was annotated to 3-indolepropionic acid, NEG1179 was annotated to indoleacrylic acid, and POS3568 was annotated to Met-Tyr.

A

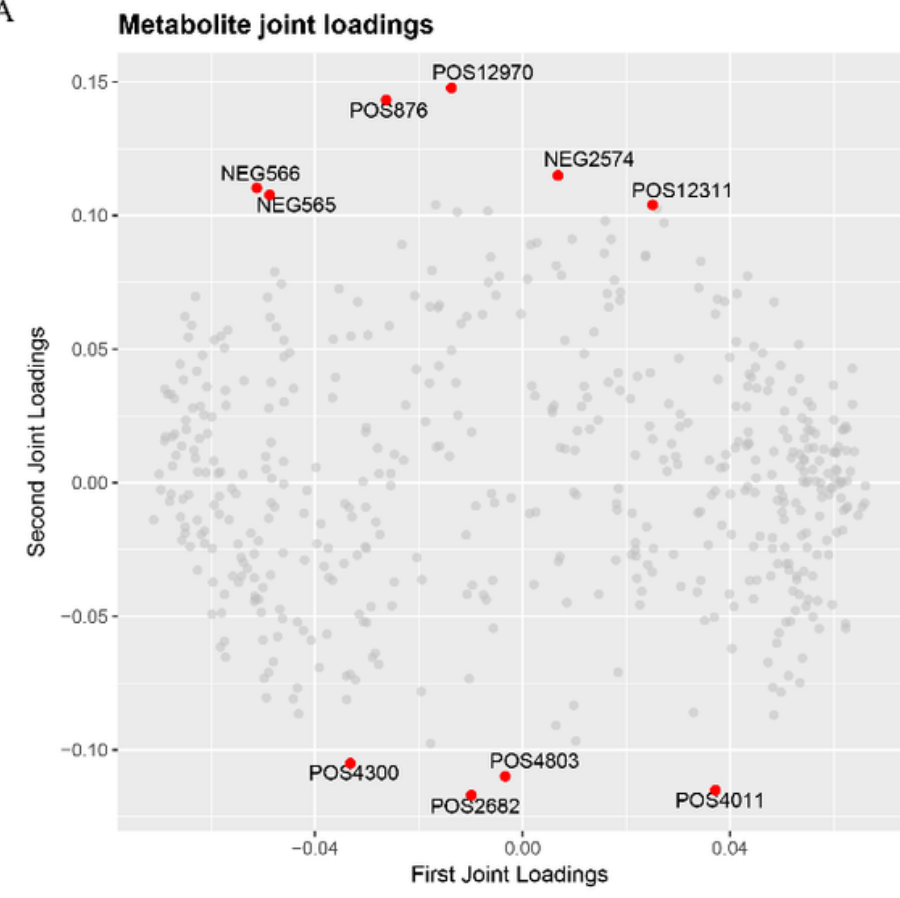

C

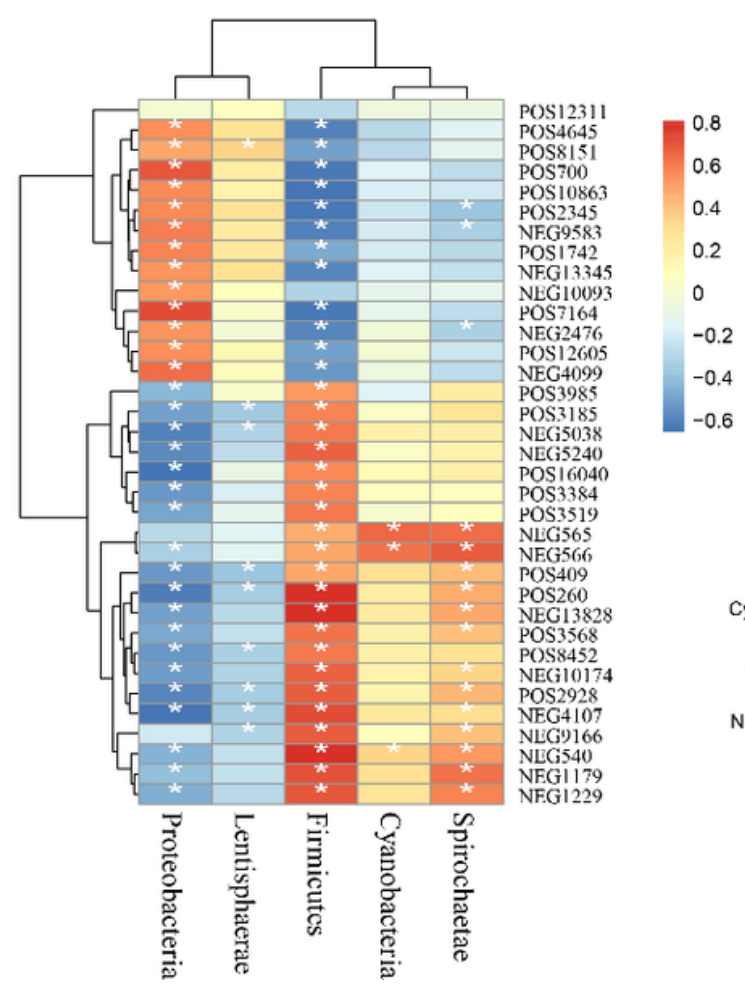

B

Phylum joint loadings

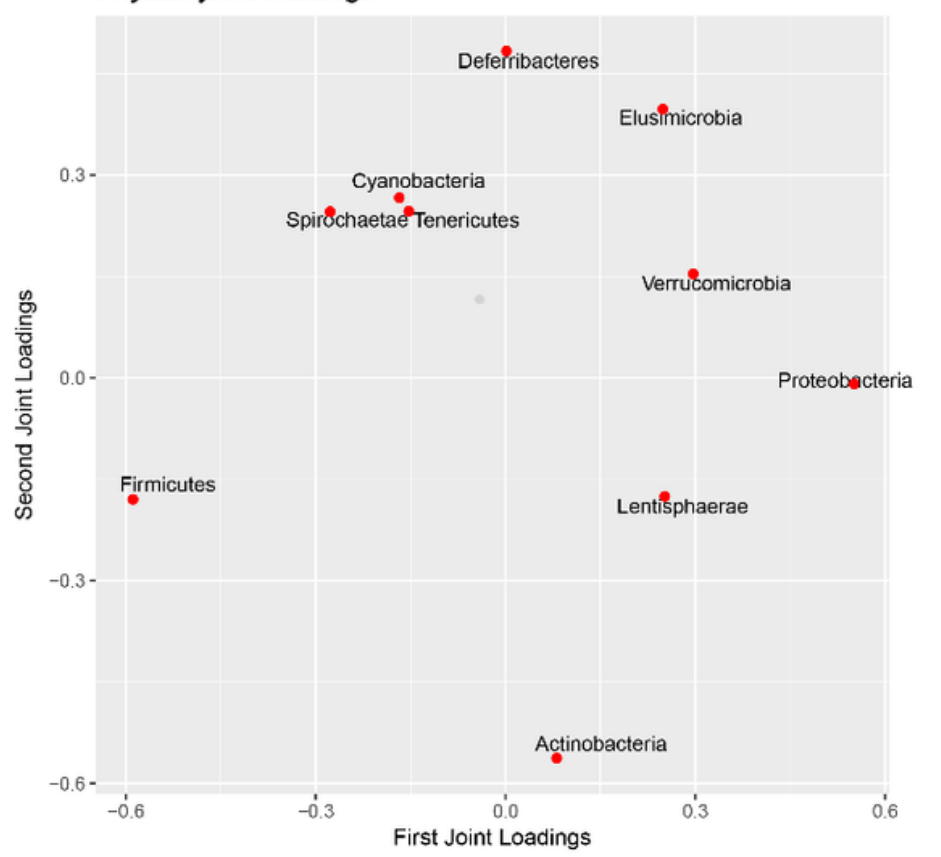

D
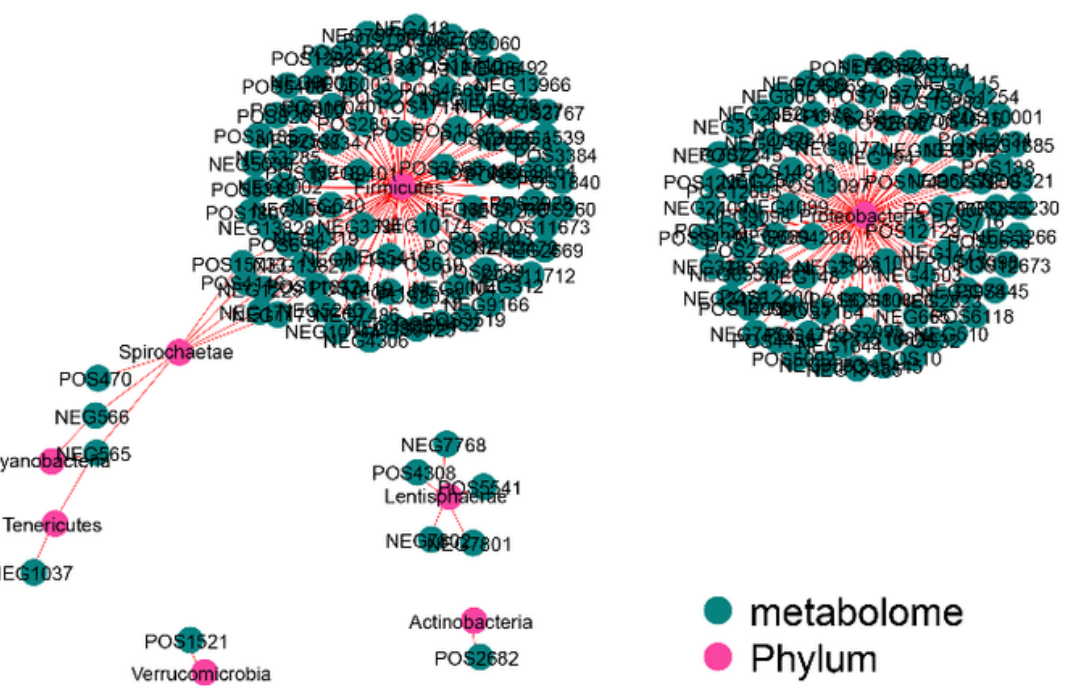

metabolome

Phylum

\section{Figure 5}

Correlations between the gut microbiome and metabolites. (A and B) Joint loading plot of O2PLS model analysis integrating metabolomics and microbiome data. (C) Heatmap of correlations between phyla and altered metabolites. (D) The correlation network of metabolome and phyla. 


\section{Supplementary Files}

This is a list of supplementary files associated with this preprint. Click to download.

- FigureS2.pdf

- Supplementarytable5diffPOSmetabolies.xls

- FigureS5.pdf

- Supplementarytable4diffNEGmetabolies.xls

- Supplementary.docx

- FigureS3.pdf

- FigureS4.pdf

- ARRIVEGuidelinesChecklistGe.pdf

- FigureS6.pdf

- FigureS1.pdf 